\title{
Knowledge Gaps in Mobile Health Research for Promoting Physical Activity in Adults With Autism Spectrum Disorder
}

OPEN ACCESS

Edited by:

Francisco José García-Peñalvo, University of Salamanca, Spain

Reviewed by:

Xiaoyi Hu,

Beijing Normal University, China Giovanni Ottoboni,

University of Bologna, Italy

${ }^{*}$ Correspondence:

Daehyoung Lee

lee03284@d.umn.edu

Specialty section:

This article was submitted to

Health Psychology,

a section of the journal

Frontiers in Psychology

Received: 29 November 2020 Accepted: 01 March 2021

Published: 24 March 2021

Citation:

Lee D (2021) Knowledge Gaps in

Mobile Health Research for

Promoting Physical Activity in Adults

With Autism Spectrum Disorder.

Front. Psychol. 12:635105.

doi: 10.3389/fpsyg.2021.635105

\section{Daehyoung Lee*}

Department of Applied Human Sciences, University of Minnesota Duluth, Duluth, MN, United States

A growing body of research highlights that adults with autism spectrum disorder (ASD) have poor health outcomes, yet effective health interventions are lacking for this population. While mobile health applications demonstrate potential for promoting physical activity (PA) in adults with ASD, scientific evidence for supporting this tool's long-term effectiveness on PA behavior change remains inconclusive. This study aimed to provide the latest information on PA research and the prospective role of mobile health applications for promoting PA in adults with ASD. A literature review demonstrated that a few available studies show contradictory results regarding PA levels in adults with ASD, and behavior change techniques and gamification-guided mobile health applications can be promising tactics to leverage autism's strengths and increase PA in these individuals. Optimizing design decisions based on needs analysis and user feedback is crucial to identifying and developing a sustainable mobile health intervention for PA promotion in adults with ASD.

Keywords: mHealth application, physical activity, adults with autism, behavior change techniques, gamification

\section{INTRODUCTION}

Autism spectrum disorder (ASD) is a pervasive neurodevelopmental condition characterized by social interaction and communication deficits, as well as restricted or repetitive behavior patterns and interests (American Psychiatric Association, 2013). According to a recent report from the Centers for Disease Control and Prevention (CDC)'s Autism and Developmental Disabilities Monitoring Network in 2020, the estimated prevalence of ASD has continuously increased since its first surveillance in 2007. Currently, 1 in 54 children in the United States have been identified with ASD, a nearly $10 \%$ increase from a previous estimate of 1 in 59 children reported in 2018 (Baio et al., 2018; Maenner, 2020). A recent modeling study conducted by Dietz et al. (2020) provided the first surveillance data on the estimated prevalence of adults with ASD in the United States using multiple national data sets. The study estimated that approximately $2.21 \%$ of United States adults (18 years and older) were living with ASD in 2017, and the prevalence was higher in adult males than females, as consistent with previous findings on children with ASD (Baio et al., 2018; Kogan et al., 2018). As remarked in the study, however, research on autism in adulthood is still a challenge because psychometrically validated tests for screening adults with ASD are currently unavailable (Dietz et al., 2020). Additionally, those who live independently with typical cognitive function may not be accurately monitored due 
to a lack of connection with public or community settings (e.g., schools, therapeutic entities, etc.), as well as an absence of surveillance systems to address this issue (Dietz et al., 2020).

Despite the relatively little attention paid towards adults with ASD, research on risk factors for developing autism and understanding the condition's impact on health outcomes has increased significantly over the past two decades (Wolff, 2004; Pellicano et al., 2014). Importantly, the existing literature has been consistent in showing that adults with ASD have poorer outcomes across health domains than those without ASD. Croen et al. (2015) studied a large sample of adults with $\operatorname{ASD}(n=1,507)$ in the United States and highlighted that these individuals are at a higher risk for nearly all mental and physical health conditions (e.g., depression, anxiety, obesity, and diabetes) compared to adults without any ASD diagnoses. Furthermore, adults with ASD are more than twice as likely as their neurotypical counterparts to die prematurely (Hirvikoski et al., 2016). It is predicted that the shortage of resources and the existing health disparities for this population will likely expand (Tyler et al., 2011; Krahn et al., 2015). This descriptive mini review aimed to provide the latest information on physical activity (PA) research and the prospective role of mobile health applications for promoting PA in adults with ASD. Four electronic databases (Google Scholar, PubMed, Scopus, and Web of Science) were searched for studies published between October 2000 and September 2020. Search terms included "physical activity," "mobile application," "autism," and a combination of these keywords and close variants (e.g., Asperger's syndrome, mHealth, fitness app, etc.). The search was limited to articles published in English.

\section{PA IN ADULTS WITH ASD}

There is little information on PA as a preventive health behavior in adults with ASD (Reinders et al., 2019). Most research in this area has been conducted in children and adolescents, and the findings indicate that they are less physically active (Pan, 2008; Stanish et al., 2017) or engage in similar levels of PA participation as their typically developing peers (Sandt and Frey, 2005; Bandini et al., 2013). More importantly, moderate to vigorous PA (MVPA), which is the level of activity associated with health outcomes, significantly decreases with age in this population (MacDonald et al., 2011). Nonetheless, PA has been used with moderate success as a social or behavioral intervention in young individuals with ASD (Sowa and Meulenbroek, 2012; Schmitz Olin et al., 2017). A previous systemic review found that PA or exercise may be effective at reducing negative behaviors (e.g., aggression, elopement, and stereotypy) and increasing positive behaviors (e.g., academic responding, on-task behavior, and motor performance) in both children and young adults with ASD (Lang et al., 2010), although the relatively small sample $(n=64)$ and varying methodological quality in existing studies make it difficult to draw a solid conclusion about the effects of PA on behavioral outcomes in this population.

National and international health organizations recommend that adults should participate in at least $150 \mathrm{~min}$ of moderateintensity PA or 75 min of vigorous-intensity PA throughout a week, or an equivalent combination of MVPA, to see health benefits (Piercy et al., 2018; World Health Organization, 2019). While the benefits of regular PA (e.g., improvement in muscular and cardiorespiratory fitness, reduced risk of cardiovascular diseases and mental disorders, and weight control) have been supported by numerous studies (Warburton et al., 2006; Warburton and Bredin, 2017), insufficient PA or physical inactivity is a leading risk factor for coronary heart disease and global premature mortality (Autenrieth et al., 2011; Lee et al., 2012). To date, there exist only a few available studies on PA in adults with ASD, and they have shown contradictory results (Eaves and Ho, 2008; Lalonde et al., 2014; Garcia-Pastor et al., 2019). Eaves and Ho (2008) used parent proxy reports and found that adults with ASD engaged in MVPA on average once per week and spent about $13 \mathrm{~h} /$ day sitting. Lalonde et al. (2014) were the first to use pedometers as an objective measure to promote walking activities in a small sample of adults with severe ASD. A walking program, along with goal setting, feedback on performance, and reinforcement, was effective at increasing daily steps to a desired level (e.g., >10,000 steps/day) in all participants. As a pilot surveillance study, Garcia-Pastor et al. (2019) used uniaxial accelerometry and found that Spanish adults with ASD met the recommended PA guidelines of $\geq 150 \mathrm{~min}$ of MVPA a week, but they were also highly sedentary. It is important to note that, however, sample characteristics in these studies were described as having moderate to severe autism symptoms and relatively low levels of cognitive function, needing high levels of support. Most of the study participants attended segregated schools or lived with parents, in supported group homes, or foster care. Moreover, the methodological heterogeneity in PA assessment (i.e., proxy survey reports vs. objective measures) makes it difficult to synthesize previous findings. As such, it is inconclusive how PA is associated with varying levels of cognitive function and the concordance between subjective and objective measures for PA assessment in adults with ASD.

\section{MOBILE HEALTH APPLICATIONS FOR PEOPLE WITH ASD}

Mobile application is an attractive modality for behavioral intervention in people with ASD because human-technology interaction enables visuospatial learning, which is often noted as a particular strength in those with ASD (Samson et al., 2012). It also presents a lower social burden compared to traditional face-to-face interaction, an area in which those with ASD struggle, as technology provides a predictable and consistent social platform (Kientz et al., 2013). With the increased accessibility and affordability, the use of mobile applications for people with ASD has been widespread across a range of domains focusing on visual cues as a supplementary intervention. Autism-friendly mobile applications are particularly designed to accommodate the unique characteristics of autistic users (e.g., avoidance of social situations and strengths in logical reasoning; American Psychiatric Association, 2013; Shah et al., 2016). A number of user-centered educational applications have gained popularity in the autism community by providing 
leaning opportunities for social and communication skills, emotional regulation skills, and occupational functioning (e.g., appropriate behavior in the workplace or school and problem-solving skills; Ayres et al., 2013; Hourcade et al., 2013; Gay and Leijdekkers, 2014).

However, there exist almost no similar attempts to use mobile applications to address PA disparities in individuals with ASD. The only related effort has been a video modeling mobile application, Exercise Buddy, that was developed to help children with ASD acquire and improve fundamental motor skills consisting of locomotor (e.g., walking, running, and jumping), balance, and object control skills (Bittner et al., 2017). With supportive visual and auditory instructions, this video modeling program effectively increased heart rate and energy expenditure in children with ASD while they performed locomotor skill tasks (Bittner et al., 2017). However, there was no post hoc investigation on the functional outcomes in relation to actual motor skill acquisition/improvement after the intervention and any consequent impact on PA or other daily living activities in real-world settings. In addition, the system focused on children with limited cognitive function and required substantial assistance for proper execution; thus, it cannot be applicable to adults with ASD who live independently and make their own choices in their health behaviors.

Although the evidence supporting their long-term effectiveness on health behavior change is unclear, mobile health applications can have small to moderate effects in the short term (e.g., up to 3 months) on self-monitoring or changing PA and sedentary behavior in overweight and sedentary neurotypical adults (TurnerMcGrievy et al., 2013; Romeo et al., 2019). PA in adults with ASD may be best stimulated by using gamified mobile applications, particularly considering reports that adults with ASD use technology devices over $4 \mathrm{~h}$ /day, primarily playing games (Lee et al., 2018). Gamified behavioral interventions have rapidly expanded the technological potential to monitor and improve PA participation in a playful way (Zuckerman and Gal-Oz, 2014; Alahäivälä and Oinas-Kukkonen, 2016), which leverages the merits of a heightened sense of autonomy, as well as feelings of fun and continuation desire (Deterding et al., 2011; King et al., 2013). Also, an emerging body of research suggests that behavior change techniques (BCTs)-guided mobile health interventions can be useful to increase PA in inactive or sedentary individuals (Direito et al., 2017; Sullivan and Lachman, 2017). The importance of incorporating evidence-based BCTs should be amplified when designing health behavior change interventions for those with ASD, considering their restricted repertoires of behavior patterns and low social motivation (Dawson et al., 2005; Chevallier et al., 2012).

\section{BCTS AND GAMIFICATION FOR PA PROMOTION IN ADULTS WITH ASD}

BCTs are a systematic, evidence-based health behavior change strategy for altering or redirecting causal processes that regulate healthy behaviors (Abraham and Michie, 2008; Michie et al., 2013). For example, some intervention strategies can be designed to monitor and promote PA participation, smoking abstinence, a healthy diet, or consistent sleep patterns by augmenting facilitators and/or mitigating barriers for behavior change (Carey et al., 2019). Since Abraham and Michie (2008) structured the first cross-behavior taxonomy of 22 BCTs, this strategy has been extensively adopted across the globe to design, implement, and evaluate behavior change interventions. On the basis of a systematic review and refinement, a current taxonomy of the BCTs includes 93 hierarchically clustered techniques, including self-monitoring of behavior, social support, goal setting, feedback on healthy behavior, action planning, incentives, etc., that offer multidisciplinary acceptance and use for developing effective behavior change interventions (Michie et al., 2013).

Effective behavior change interventions generally leverage multiple BCTs to implement health behavior change, and a mobile application enables researchers to deliver diverse BCTs in a time- and cost-effective way in real-world settings (Bird et al., 2013; Middelweerd et al., 2014; Crane et al., 2015). Currently, there are more than 325,000 health applications specifically designed for promoting physical fitness and PA in major application stores such as Google Play and the Apple App Store (Research2Guidance, 2017). Some of the available applications have empirically demonstrated the potential to enhance users' PA engagement, but only a minimal number of the applications have been developed or evaluated through scientific methods, while most are not guided by evidence-based health intervention strategies such as BCTs (Cowan et al., 2013; Pagoto and Bennett, 2013).

Conroy et al. (2014) examined 167 top-ranked PA-promoting applications on the commercial market to verify the prevalence of BCTs among the popular PA applications. Specifically, they characterized the BCTs by analyzing the applications' online descriptions and functions on the basis of the BCTs taxonomy. Among others, provision of instruction for behavior performance, demonstration of behavior, provision of contingent feedback on performance, behavioral goal-setting, and plans of social support or change were the most commonly adopted BCTs in the included applications (Conroy et al., 2014; Yang et al., 2015). Importantly, the majority of contemporary PA-promoting applications lacked BCTs as a theoretical background for the developmental process (Conroy et al., 2014). Due to the scarcity of systematic assessment within the contemporary PA applications, it is unclear how the incorporated BCTs affected and changed users' health behavior, as well as how any positive health outcomes after the application use were associated with varying intrinsic or extrinsic motivation factors (Sullivan and Lachman, 2017).

Despite the limitations, gamified behavior change interventions in particular have presented promising results to promote PA in adults with and without ASD. Gamified interventions leverage game-like elements such as leaderboards, animated avatars, problem-solving, and attractive storylines to elevate the level of motivation and user engagement (Alsawaier, 2018). Chen and $\mathrm{Pu}$ (2014) deployed "HealthyTogether," an interactive mobile fitness application, which focused on gamified social interaction among users leveraging social incentives and a point reward system. The research team found a significant relationship between 
$\mathrm{PA}$ and the number of messages that participants exchanged, implying the importance of cooperative social interaction in gamified PA contexts. The success of Pokémon Go, an augmented reality-based application that links a Pokémon character hunting game to location tracking technology, further heightened the emerging potential of using gamification techniques to promote achievable PA (e.g., walking) in real-life settings, even though the intervention effect was short-term (Althoff et al., 2016; Howe et al., 2016). Furthermore, Lee (2020) reported that "PuzzleWalk" a gamified, BCTs-based mobile application, can be effective at increasing user engagement and potentially PA in adults with high-functioning ASD. Their preliminary deployment study compared PuzzleWalk to a commercially popular PA-tracking application, Google Fit, to evaluate the efficacy of both apps on increasing PA in adults with ASD who do not have intellectual disability, but struggle with daily living skills using a randomized controlled trial design $(n=24)$. Findings indicated that PuzzleWalk users spent significantly larger amounts of time on application use compared to Google Fit users throughout a 5-week intervention period. Even though statistical significance was not reached, PuzzleWalk also demonstrated the potential for increasing MVPA in the short term and reducing sedentary time in adults with high-functioning ASD (Lee, 2020).

Taken together, a growing body of research supports the potential of gamification and BCTs-guided mobile applications for promoting PA in diverse population groups, including adults with ASD. However, despite the increasing popularity of using these promising tactics in commercial PA applications, evidencebased mobile health interventions are lacking, and only a few attempts have been made to address the unique characteristics and health needs in adults with ASD. Furthermore, more research is warranted to better understand the impact of varying degrees of cognitive abilities and sociability on health behaviors in adults with ASD.

\section{CONCLUDING REMARKS}

This brief review has revealed that there is a critical need for interventional studies that leverage autism's strengths to address preventive health and reduce health disparities in adults with ASD. Despite the seemingly successful expansion of mobile

\section{REFERENCES}

Abraham, C., and Michie, S. (2008). A taxonomy of behavior change techniques used in interventions. Health Psychol. 27, 379-387. doi: 10.1037/0278-6133.27.3.379

Alahäivälä, T., and Oinas-Kukkonen, H. (2016). Understanding persuasion contexts in health gamification: a systematic analysis of gamified health behavior change support systems literature. Int. J. Med. Inform. 96, 62-70. doi: 10.1016/j.ijmedinf.2016.02.006

Alsawaier, R. S. (2018). The effect of gamification on motivation and engagement. Int. J. Inf. Learn. Technol. 35, 56-79. doi: 10.1108/IJILT-02-2017-0009

Althoff, T., White, R. W., and Horvitz, E. (2016). Influence of Pokémon go on physical activity: study and implications. J. Med. Internet Res. 18:e315. doi: 10.2196/jmir.6759

American Psychiatric Association (2013). Diagnostic and statistical manual of mental disorders (DSM-5). 5th Edn. Arlington, VA: American Psychiatric Association. health applications for promoting PA in both adults with and without ASD, most of the existing health and PA applications on the commercial market are not designed using scientific evidence or health behavior theories such as BCTs (Middelweerd et al., 2014; Yang et al., 2015). It is also crucial to note that reports on the success of gamified applications are largely based on anecdotal evidence, and there is insufficient scientific evidence to support their long-term effectiveness in inducing voluntary behavior change, including PA engagement (King et al., 2013; Lister et al., 2014). The use of gamification principles in most popular PA applications does not adhere to any industrystandard or professional guidelines (Lister et al., 2014). Although BCTs and gamification are promising tactics for increasing user interest and PA motivation, it is inconclusive whether these features can lead to sustained behavior change. Furthermore, due to an absence of versatility in addressing the unique behavioral and social preferences of individuals with ASD (e.g., adherence to routines and visual preference for social stimuli; Leekam et al., 2011; Crawford et al., 2016), general mobile health applications are less likely to enact a successful PA behavior change in adults with ASD who tend to have relatively low self-esteem and intrinsic motivation for health behavior change (Koegel and Mentis, 1985; Cooper et al., 2017). To develop a sustainable mobile health intervention that promotes PA in adults with ASD, it is critical to optimize design decisions based on an in-depth understanding of the target users and iterative inquiry regarding user feedback and behavior patterns (i.e., user-centered design) to facilitate the use of and adherence to interventions (Mummah et al., 2016; Lee et al., 2020).

\section{AUTHOR CONTRIBUTIONS}

The author confirms being the sole contributor of this work and has approved it for publication.

\section{FUNDING}

This research was supported by the ACSM Foundation Doctoral Student Research Grant from the American College of Sports Medicine Foundation (\#19-01155). 
spectrum disorders and typically developing children. Autism 17, 44-54. doi: $10.1177 / 1362361312437416$

Bird, E. L., Baker, G., Mutrie, N., Ogilvie, D., Sahlqvist, S., and Powell, J. (2013). Behavior change techniques used to promote walking and cycling: a systematic review. Health Psychol. 32, 829-838. doi: 10.1037/a0032078

Bittner, M. D., Rigby, B. R., Silliman-French, L., Nichols, D. L., and Dillon, S. R. (2017). Use of technology to facilitate physical activity in children with autism spectrum disorders: a pilot study. Physiol. Behav. 177, 242-246. doi: 10.1016/j.physbeh.2017.05.012

Carey, R. N., Connell, L. E., Johnston, M., Rothman, A. J., de Bruin, M., Kelly, M. P., et al. (2019). Behavior change techniques and their mechanisms of action: a synthesis of links described in published intervention literature. Ann. Behav. Med. 53, 693-707. doi: 10.1093/abm/kay078

Chen, Y., and $\mathrm{Pu}, \mathrm{P}$. (2014). "HealthyTogether: exploring social incentives for mobile fitness applications" in Proceedings of the Second International Symposium of Chinese-CHI; April 2014; ACM, 25-34.

Chevallier, C., Kohls, G., Troiani, V., Brodkin, E. S., and Schultz, R. T. (2012). The social motivation theory of autism. Trends Cogn. Sci. 16, 231-239. doi: 10.1016/j.tics.2012.02.007

Conroy, D. E., Yang, C. H., and Maher, J. P. (2014). Behavior change techniques in top-ranked mobile apps for physical activity. Am. J. Prev. Med. 46, 649-652. doi: 10.1016/j.amepre.2014.01.010

Cooper, K., Smith, L. G. E., and Russell, A. (2017). Social identity, self-esteem, and mental health in autism. Eur. J. Soc. Psychol. 47, 844-854. doi: 10.1002/ ejsp.2297

Cowan, L. T., Van Wagenen, S. A., Brown, B. A., Hedin, R. J., Seino-Stephan, Y., Hall, P. C., et al. (2013). Apps of steel: are exercise apps providing consumers with realistic expectations? A content analysis of exercise apps for presence of behavior change theory. Health Educ. Behav. 40, 133-139. doi: $10.1177 / 1090198112452126$

Crane, D., Garnett, C., Brown, J., West, R., and Michie, S. (2015). Behavior change techniques in popular alcohol reduction apps: content analysis. J. Med. Internet Res. 17:e118. doi: 10.2196/jmir.4060

Crawford, H., Moss, J., Oliver, C., Elliott, N., Anderson, G. M., and McCleery, J. P. (2016). Visual preference for social stimuli in individuals with autism or neurodevelopmental disorders: an eye-tracking study. Mol. Autism 7:24. doi: 10.1186/s13229-016-0084-x

Croen, L. A., Zerbo, O., Qian, Y., Massolo, M. L., Rich, S., Sidney, S., et al. (2015). The health status of adults on the autism spectrum. Autism 19, 814-823. doi: $10.1177 / 1362361315577517$

Dawson, G., Webb, S. J., and McPartland, J. (2005). Understanding the nature of face processing impairment in autism: insights from behavioral and electrophysiological studies. Dev. Neuropsychol. 27, 403-424. doi: 10.1207/ s15326942dn2703_6

Deterding, S., Dixon, D., Khaled, R., and Nacke, L. (2011). "From game design elements to gamefulness: defining gamification" in Proceedings of the 15th International Academic MindTrek Conference: Envisioning Future Media Environments; September 2011; 9-11.

Dietz, P. M., Rose, C. E., McArthur, D., and Maenner, M. (2020). National and state estimates of adults with autism spectrum disorder. J. Autism Dev. Disord. 50, 4258-4266. doi: 10.1007/s10803-020-04494-4

Direito, A., Carraça, E., Rawstorn, J., Whittaker, R., and Maddison, R. (2017). mHealth technologies to influence physical activity and sedentary behaviors: behavior change techniques, systematic review and meta-analysis of randomized controlled trials. Ann. Behav. Med. 51, 226-239. doi: 10.1007/ s12160-016-9846-0

Eaves, L. C., and Ho, H. H. (2008). Young adult outcome of autism spectrum disorders. J. Autism Dev. Disord. 38, 739-747. doi: 10.1007/s10803-007-0441-x

Garcia-Pastor, T., Salinero, J. J., Theirs, C. I., and Ruiz-Vicente, D. (2019). Obesity status and physical activity level in children and adults with autism spectrum disorders: a pilot study. J. Autism Dev. Disord. 49, 165-172. doi: 10.1007/s10803-018-3692-9

Gay, V., and Leijdekkers, P. (2014). Design of emotion-aware mobile apps for autistic children. Heal. Technol. 4, 21-26. doi: 10.1007/s12553-013-0066-3

Hirvikoski, T., Mittendorfer-Rutz, E., Boman, M., Larsson, H., Lichtenstein, P., and Bölte, S. (2016). Premature mortality in autism spectrum disorder. $B r$. J. Psychiatry 208, 232-238. doi: 10.1192/bjp.bp.114.160192

Hourcade, J. P., Williams, S. R., Miller, E. A., Huebner, K. E., and Liang, L. J. (2013). "Evaluation of tablet apps to encourage social interaction in children with autism spectrum disorders" in Proceedings of the SIGCHI Conference on Human Factors in Computing Systems-CHI'13; April 2013; 3197-3206.

Howe, K. B., Suharlim, C., Ueda, P., Howe, D., Kawachi, I., and Rimm, E. B. (2016). Gotta catch'em all! Pokémon GO and physical activity among young adults: difference in differences study. BMJ 355:i6270. doi: 10.1136/bmj. i 6270

Kientz, J. A., Goodwin, M. S., Hayes, G. R., and Abowd, G. D. (2013). "Interactive technologies for autism" in Synthesis lectures on assistive, rehabilitative, and health-preserving technologies. Vol. 2. 1-177.

King, D., Greaves, F., Exeter, C., and Darzi, A. (2013). “Gamification”: influencing health behaviours with games. J. R. Soc. Med. 106, 76-78. doi: $10.1177 / 0141076813480996$

Koegel, R. L., and Mentis, M. (1985). Motivation in childhood autism: can they or won't they? J. Child Psychol. Psychiatry 26, 185-191. doi: 10.1111/ j.1469-7610.1985.tb02259.x

Kogan, M. D., Vladutiu, C. J., Schieve, L. A., Ghandour, R. M., Blumberg, S. J., Zablotsky, B., et al. (2018). The prevalence of parent-reported autism spectrum disorder among US children. Pediatrics 142:e20174161. doi: 10.1542/ peds.2017-4161

Krahn, G. L., Walker, D. K., and Correa-De-Araujo, R. (2015). Persons with disabilities as an unrecognized health disparity population. Am. J. Public Health 105, S198-S206. doi: 10.2105/AJPH.2014.302182

Lalonde, K. B., MacNeill, B. R., Eversole, L. W., Ragotzy, S. P., and Poling, A. (2014). Increasing physical activity in young adults with autism spectrum disorders. Res. Autism Spectr. Disord. 8, 1679-1684. doi: 10.1016/j. rasd.2014.09.001

Lang, R., Koegel, L. K., Ashbaugh, K., Regester, A., Ence, W., and Smith, W. (2010). Physical exercise and individuals with autism spectrum disorders: a systematic review. Res. Autism Spectr. Disord. 4, 565-576. doi: 10.1016/j. rasd.2010.01.006

Lee, D. (2020). Preliminary efficacy of a gamified, behavior change techniquesbased mobile application Puzzlewalk on increasing physical activity and reducing anxiety in adults with autism spectrum disorder. doctoral dissertation. Indiana University, ProQuest Dissertations Publishing.

Lee, D., Frey, G. C., Cheng, A., and Shih, P. C. (2018). "Puzzle walk: a gamified mobile app to increase physical activity in adults with autism spectrum disorder" in Prodeedings of the 2018 10th International Conference on Virtual Worlds and Games for Serious Applications (VS-Games); September 2018; $1-4$.

Lee, D., Frey, G. C., Min, A., Kim, B., Cothran, D. J., Bellini, S., et al. (2020). Usability inquiry of a gamified behavior change app for increasing physical activity and reducing sedentary behavior in adults with and without autism spectrum disorder. Health Inform. J. 26, 2992-3008. doi: 10.1177/14604 58220952909

Lee, I. M., Shiroma, E. J., Lobelo, F., Puska, P., Blair, S. N., Katzmarzyk, P. T. et al. (2012). Effect of physical inactivity on major non-communicable diseases worldwide: an analysis of burden of disease and life expectancy. Lancet 380, 219-229. doi: 10.1016/S0140-6736(12)61031-9

Leekam, S. R., Prior, M. R., and Uljarevic, M. (2011). Restricted and repetitive behaviors in autism spectrum disorders. Psychol. Bull. 137, 562-593. doi: 10.1037/a0023341

Lister, C., West, J. H., Cannon, B., Sax, T., and Brodegard, D. (2014). Just a fad? Gamification in health and fitness apps. J. Med. Internet Res. 16:e9. doi: 10.2196/games.3413

MacDonald, M., Esposito, P., and Ulrich, D. (2011). The physical activity patterns of children with autism. BMC Res. Notes 4:422. doi: 10.1186/1756-0500-4-422

Maenner, M. J. (2020). Prevalence of autism spectrum disorder among children aged 8 years-autism and developmental disabilities monitoring network, 11 sites, United States, 2016. MMWR Surveill. Summ. 69, 1-12. doi: 10.15585/ mmwr.ss6904al

Michie, S., Richardson, M., Johnston, M., Abraham, C., Francis, J., Hardeman, W. et al. (2013). The behavior change technique taxonomy (v1) of 93 hierarchically clustered techniques: building an international consensus for the reporting of behavior change interventions. Ann. Behav. Med. 46, 81-95. doi: 10.1007/ s12160-013-9486-6

Middelweerd, A., Mollee, J. S., van der Wal, C. N., Brug, J., and te Velde, S. J. (2014). Apps to promote physical activity among adults: a review and content analysis. Int. J. Behav. Nutr. Phys. Act. 11:97. doi: 10.1186/ s12966-014-0097-9 
Mummah, S. A., Robinson, T. N., King, A. C., Gardner, C. D., and Sutton, S. (2016). IDEAS (integrate, design, assess, and share): a framework and toolkit of strategies for the development of more effective digital interventions to change health behavior. J. Med. Internet Res. 18:e317. doi: 10.2196/jmir.5927

Pagoto, S., and Bennett, G. G. (2013). How behavioral science can advance digital health. Transl. Behav. Med. 3, 271-276. doi: 10.1007/s13142-013-0234-z

Pan, C.-Y. (2008). Objectively measured physical activity between children with autism spectrum disorders and children without disabilities during inclusive recess settings in Taiwan. J. Autism Dev. Disord. 38, 1292-1301. doi: 10.1007/ s10803-007-0518-6

Pellicano, E., Dinsmore, A., and Charman, T. (2014). What should autism research focus upon? Community views and priorities from the United Kingdom. Autism 18, 756-770. doi: 10.1177/1362361314529627

Piercy, K. L., Troiano, R. P., Ballard, R. M., Carlson, S. A., Fulton, J. E., Galuska, D. A., et al. (2018). The physical activity guidelines for Americans. JAMA 320, 2020-2028. doi: 10.1001/jama.2018.14854

Reinders, N. J., Branco, A., Wright, K., Fletcher, P. C., and Bryden, P. J. (2019). Scoping review: physical activity and social functioning in young people with autism spectrum disorder. Front. Psychol. 10:120. doi: 10.3389/fpsyg.2019.00120

Research2Guidance (2017). mHealth app economics 2017: current status and future trends in mobile health. Available at: https://research2guidance.com/ product/mhealth-economics-2017-current-status-and-future-trends-in-mobilehealth/ (Accessed May 1, 2019).

Romeo, A., Edney, S., Plotnikoff, R., Curtis, R., Ryan, J., Sanders, I., et al. (2019). Can smartphone apps increase physical activity? Systematic review and meta-analysis. J. Med. Internet Res. 21, 1-14. doi: 10.2196/12053

Samson, F., Mottron, L., Soulières, I., and Zeffiro, T. A. (2012). Enhanced visual functioning in autism: an ALE meta-analysis. Hum. Brain Mapp. 33, 1553-1581. doi: 10.1002/hbm.21307

Sandt, D. D. R., and Frey, G. C. (2005). Comparison of physical activity levels between children with and without autistic spectrum disorders. Adapt. Phys. Act. Q. 22, 146-159. doi: 10.1123/apaq.22.2.146

Schmitz Olin, S., McFadden, B. A., Golem, D. L., Pellegrino, J. K., Walker, A. J., Sanders, D. J., et al. (2017). The effects of exercise dose on stereotypical behavior in children with autism. Med. Sci. Sports Exerc. 49, 983-990. doi: 10.1249/MSS.0000000000001197

Shah, P., Catmur, C., and Bird, G. (2016). Emotional decision-making in autism spectrum disorder: the roles of interoception and alexithymia. Mol. Autism 7:43. doi: 10.1186/s13229-016-0104-x

Sowa, M., and Meulenbroek, R. (2012). Effects of physical exercise on autism spectrum disorders: a meta-analysis. Res. Autism Spectr. Disord. 6, 46-57. doi: 10.1016/j.rasd.2011.09.001

Stanish, H. I., Curtin, C., Must, A., Phillips, S., Maslin, M., and Bandini, L. G. (2017). Physical activity levels, frequency, and type among adolescents with and without autism spectrum disorder. J. Autism Dev. Disord. 47, 785-794. doi: 10.1007/s10803-016-3001-4

Sullivan, A. N., and Lachman, M. E. (2017). Behavior change with fitness technology in sedentary adults: a review of the evidence for increasing physical activity. Front. Public Health 4:289. doi: 10.3389/fpubh.2016.00289

Turner-McGrievy, G. M., Beets, M. W., Moore, J. B., Kaczynski, A. T., Barr-Anderson, D. J., and Tate, D. F. (2013). Comparison of traditional versus mobile app self-monitoring of physical activity and dietary intake among overweight adults participating in an mHealth weight loss program. J. Am. Med. Inform. Assoc. 20, 513-518. doi: 10.1136/amiajnl-2012001510

Tyler, C. V., Schramm, S. C., Karafa, M., Tang, A. S., and Jain, A. K. (2011). Chronic disease risks in young adults with autism spectrum disorder: forewarned is forearmed. Am. J. Intellect. Dev. Disabil. 116, 371-380. doi: 10.1352/1944-7558-116.5.371

Warburton, D. E. R., and Bredin, S. S. D. (2017). Health benefits of physical activity: a systematic review of current systematic reviews. Curr. Opin. Cardiol. 32, 541-556. doi: 10.1097/HCO.0000000000000437

Warburton, D. E. R., Nicol, C. W., and Bredin, S. S. D. (2006). Health benefits of physical activity: the evidence. Can. Med. Assoc. J. 174, 801-809. doi: 10.1503/cmaj.051351

Wolff, S. (2004). The history of autism. Eur. Child Adolesc. Psychiatry 13, 201-208. doi: 10.1007/s00787-004-0363-5

World Health Organization (2019). Global action plan on physical activity 2018-2030: More active people for a healthier world. Geneva, Switzerland: World Health Organization.

Yang, C.-H., Maher, J. P., and Conroy, D. E. (2015). Implementation of behavior change techniques in mobile applications for physical activity. Am. J. Prev. Med. 48, 452-455. doi: 10.1016/j.amepre.2014.10.010

Zuckerman, O., and Gal-Oz, A. (2014). Deconstructing gamification: evaluating the effectiveness of continuous measurement, virtual rewards, and social comparison for promoting physical activity. Pers. Ubiquit. Comput. 18, 1705-1719. doi: 10.1007/s00779-014-0783-2

Conflict of Interest: The author declares that the research was conducted in the absence of any commercial or financial relationships that could be construed as a potential conflict of interest.

Copyright (c) 2021 Lee. This is an open-access article distributed under the terms of the Creative Commons Attribution License (CC BY). The use, distribution or reproduction in other forums is permitted, provided the original author(s) and the copyright owner(s) are credited and that the original publication in this journal is cited, in accordance with accepted academic practice. No use, distribution or reproduction is permitted which does not comply with these terms. 